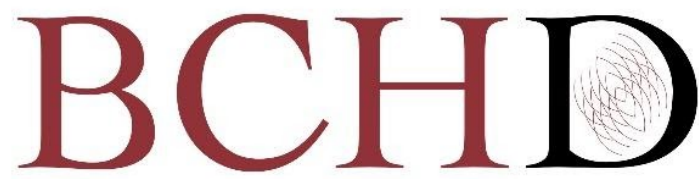

Bioactive Compounds in Health and Disease

\title{
No evidence of allergenic reactions to soy lecithin phospholipids used in Membrane Lipid Replacement studies
}

\begin{abstract}
Garth L. Nicolson ${ }^{1 *}$, Robert Settineri²
${ }^{1}$ Department of Molecular Pathology, The Institute for Molecular Medicine, Huntington Beach, California 92649, USA; ${ }^{2}$ Sierra Productions Research, Mission Viejo, California 92692, USA

*Corresponding author: Prof. Emeritus Garth L. Nicolson, PhD, MD (H), Department of Molecular Pathology, The Institute for Molecular Medicine, P.O. Box 9355, S. Laguna Beach, CA 92652

Submission Date: December 21 ${ }^{\text {st }}$, 2020; Acceptance Date: January 21, 2021; Publication Date: January 27, 2021

Please cite this article as: Nicolson G.L., Settineri R. No evidence of allergenic reasctions to soy lecithin phospholipids used in Membrane Lipid Replacement studies. Bioactive Compounds in Health and Disease 2021. 4(1): 9-13. DOI: https://www.doi.org/10.31989/bchd.v4i1.770
\end{abstract}

ABSTRACT
Membrane Lipid Replacement (MLR)
is the functional use of dietary
supplements containing cell
membrane glycerolphospholipids and
antioxidants to safely replace and
remove damaged membrane
phospholipids that accumulate during
various chronic and acute illnesses and during aging. Some products used in MLR are obtained from soy lecithin
extracts that contain cell membrane glycerolphospholipids. Thus the soybean source has been questioned
because of concerns related to genetic engineering (GMO) and the potential presence of hormone-like
components and soy allergens. There is a complete absence of proteins, glycoproteins or carbohydrates in soy
lecithins and MLR supplements that could be allergenic. One lecithin ingredient that contains purified membrane
phospholipids (NTFactor Lipids ${ }^{\circ}$ ) has been shown to produce significant positive health benefits in clinical trials.
NTFactor Lipids are fractionated and purified from non-GMO soy lecithin, but this formulation does not contain
detectable amounts of protein or glycoprotein allergens or other components that could elicit allergic or non-
allergic adverse reactions. In addition, allergenic and non-allergenic reactions have not been found in multiple
clinical trials and studies that have utilized this dietary supplement. NTFactor Lipids are manufactured in certified
Good Manufacturing Practices (cGMP) facilities using established allergen-control strategies to minimize any cross-


contact with food allergens.

Keywords: Phospholipids, Clinical trials, Allergies, Cellular membranes

CFFC 2021. This is an Open Access article distributed under the terms of the Creative Commons Attribution 4.0 License (http://creativecommons.org/licenses/by/4.0)

\section{INTRODUCTION}

Membrane glycerolphospholipids form the matrix of all biological membranes [1]. When they are damaged by free radical oxidants during aging and disease [24], they must be repaired or replaced to retain normal cellular functions $[5,6]$. The replacement process has been termed Membrane Lipid Replacement (MLR), and this has been achieved using natural plant dietary membrane phospholipid supplements that repair cellular membranes by replacement and sequester the damaged glycerolphospholipids for their natural removal in order to maintain a variety of cellular and tissue functions and to improve general health [5-8]. Multiple studies have shown the health benefits of taking oral MLR glycerolphospholipids, and these studies have not found adverse allergenic reactions among trial participants (reviewed in $[5,6,8]$ ). Here we discuss the consequences that MLR supplements like NTFactor Lipids ${ }^{\circledR}$ do not contain allergenic components.

Soy Lecithins and MLR-Absence of Soy Allergens: Dietary MLR products like NTFactor Lipids ${ }^{\circledR}$ are obtained from selected natural soybeans that are not contaminated (non-GMO) with geneticallymodified soybeans. The fractionated soy lecithins do not contain measureable amounts of soy proteins, glycoproteins or carbohydrates (the composition of NTFactor Lipids ${ }^{\circledast}$ can be found in [5]). NTFactor Lipids ${ }^{\oplus}$ are manufactured in certified Good Manufacturing Practices (cGMP) facilities following established allergen-control strategies to minimize any cross-contact with food allergens. This manufacturing process also complies with National Sanitation Foundation (NSF) requirements to provide the highest quality phospholipid products that conforms to the Food Chemicals Codex.

Soy lecithins have been used as additives in thousands of foods and food supplements without any problems associated with soy allergies [9-12]. When these soy lecithins were studied in more detail, they were found to be allergen-free. For example, Müller et al. [12] examined commercial soy lecithins for residual allergenicity and compared them with extracts from untreated or heat-treated soybeans. They concluded that commercial soy lecithins do not contain soy proteins, do not cause soy allergenic reactions and are safe for soy-allergic consumers. Similarly, Awazuhara et al. [11] studied the antigenicity of the small amounts of residual proteins that are found in some soy lecithins and soybean oils. They found vanishingly small or no antigenicity or allergenicity in these residual proteins, as measured by the binding of $\operatorname{IgE}$ and other immunoglobulins to residual lecithin proteins and by allergic challange to the residual lecithin proteins. Thus there is no evidence indicating that soy lecithins, the starting ingredient for manufacturing MLR supplements like NTFactor Lipids ${ }^{\circledR}$, contain any soy allens that could cause reactions in soy-allergic individuals [11].

MLR Use in Clinical Studies-No Evidence of Allergenic Reactions: Membrane lipids are 
commonly used in dietary supplements for support of general health $[12,13]$, and they have also been used to improve the clinical status of chronic illness patients (reviewed in $[5,6,8]$ ). The most common therapeutic use of MLR products is to treat fatigue that is caused by loss of mitochondrial function $[5,6$, 8]. Fatigue is the most common complaint of patients seeking general medical care, and it is associated with aging and most if not all chronic medical conditions [14]. Fatigue develops in older idividuals and in chronic diseases due to different possible causes, most notibly the loss of mitochondrial function and reductions in the synthesis of ATP [15]. Using MLR NTFactor ${ }^{\circ}$ or NTFactor Lipids fatigue has been successfully and significantly reduced in hundreds of patients with chronic fatigue (fatigue lasting more than 6 months), chronic fatigue syndrome (CFS/ME), fibromyalgia, Gulf War illnesses and other fatiguing illnesses without any evidence of allergenic soy reactions (reviewed in $[5,6,8]$ ).

In addition to fatigue, MLR supplements have been used to improve mental clarity, focus and memory loss. For example, volunteers were given NTFactor Lipids ${ }^{\circ}$ dissolved in a drink, and fatigue and mental focus were examined after 3 hours. A majority of the participants responded within 1 hour, and by 3 hours perceived improvements in cognition, mental clarity and focus and fatigue reductions were found [16].

Widespread pain is another symptom treated with MLR supplements, such as NTFactor Lipids ${ }^{\circ}[17$, 18]. Pain is a complex symptom that involves nerve cell channelopathies, microcirculation, autoimmune responses and other factors $[19,20]$. In fibromyalgia patients NTFactor Lipids significantly reduced pain, fatigue, gastrointestinal symptoms and improved Quality of Life assessments without evidence of adverse reactions [17]. In patients with widespread musculoskeletal and peripheral pain, chronic fatigue and many other signs and symptoms there were dramatic improvements while taking oral NTFactor Lipids ${ }^{\bullet}[18]$.

In the multiple clinical studies cited above and in various publications cited in reviews on the subject none of the patients reported any allergenic reactions to NTFactor ${ }^{\circ}$ or NTFactor Lipids ${ }^{\circ}$ contained in various natural supplements (reviewed in $[5,6$, 8]).

\section{CONCLUSIONS}

There is no evidence that indicates that taking oral MLR supplements made from soy lecithins results in allergenic or other adverse reactions. Thus these natural food supplements are safe. The FDA has determined that lecithins are GRAS (Generally Regarded As Safe) and safe to use, even in soyallergic individuals [21, 22].

List of Abbreviations: CFS/ME: Chronic fatigue syndrome/myalgic encephalomyelitis, cGMP: Certified Good Manufacturing Practices, GMO: genetically modified organisms, GRAS: Generally Regarded As Safe, IgE: immunoglobulin E, MLR: Membran Lipid Replacement, NSF: National Science Foundation

Acknowledgements: The authors would like to thank Nutritional Therapeutics, Inc. and the Institute for Molecular Medicine for financial support and for providing study materials. 
Authors Contributions: The article was written by

Garth L. Nicolson with the input of Robert Settineri.

Competing Interests: Garth L. Nicolson is a part-time consultant to Naturally Plus Tiawan and Nutritional Therapeutics, Inc. Robert Settineri is a part-time consultant to Naturally Plus USA and Nutritional Therapeutics, Inc.

\section{REFERENCES}

1. Nicolson GL: The Fluid-Mosaic Model of Membrane Structure: still relevant to understanding the structure, function and dynamics of biological membranes after more than 40 years. Biochimica et Biophysica Acta Biomembranes 2014; 1838: 1451-1466. https://doi.org/10.1016/j.bbamem.2013.10.019

2. Knight JA: Diseases related to oxygen-derived free radicals. Annals of Clinical and Laboratory Medicine 1995; 25(2): 111-121. http://www.annclinlabsci.org/content/25/2/111.full.p df+html

3. Gutteridge JMC: Free radicals in disease processes: a complilation of cause and consequence. Free Radical Research Communications 1993; 19(3): 141-158. https://doi.org/10.3109/10715769309111598

4. Harman D: Aging: a theory based on free radical and radiation chemistry. Journal of Gerontology 1956; 11(3): 298-300. https://doi.org/10.1093/geronj/11.3.298

5. Nicolson GL: Membrane Lipid Replacement: clinical studies using a natural medicine approach to restoring membrane function and improving health. International Journal of Clinical Medicine 2016; 7: 133 143. DOI: $10.4236 / \mathrm{ijcm} .2016 .72015$

6. Nicolson GL, Ash ME: Membrane Lipid Replacement for chronic illnesses, aging and cancer using oral glycerolphospholipid formulations with fructooligosaccharides to restore phospholipid function in cellular membranes, organelles, cells and tissues. Biochimica et Biophysica Acta Biomembranes 2017; 1859: 1704-1724. http://dx.doi.org/10.1016/j.bbamem.2017.04.013

7. Küllenberg $D$, Taylor LA, Schneider M, Massing U: Health effects of dietary phospholipids. Lipids in Health and Disease 2012; 11: e3.
https://doi.org/10.1186/1476-511X-11-3

8. Nicolson $\mathrm{GL}$, Rosenblatt $\mathrm{S}$, Ferreira de Mattos $\mathrm{G}$, Settineri R, Breeding PC, Ellithorpe RR, Ash ME: Clinical uses of Membrane Lipid Replacement supplements in restoring membrane function and reducing fatigue in chronic diseases and cancer. Discoveries 2016; 4(1): e54. doi: 10.15190/d.2016.1

9. Colgan M: Lecithin does not cause allergic reactions. World Press Health \& Nutrition Articles, Feb 2012. https://colganprograms.wordpress.com/2012/02/28/I ecithin-does-not-cause-allergic-reactions/

10. Bush RK, Taylor SL, Nordlee JA, Busse WW: Soybean oil is not allergenic to soybean-sensitive individuals. Journal of Allergy and Clinical Immunology 1985; 76(2): 242-245. https://www.jacionline.org/action/showPdf?pii=00916749\%2885\%2990709-2

11. Awazuhara $\mathrm{H}$, Kawai $\mathrm{H}$, Baba $\mathrm{M}$, Matsui $\mathrm{T}$, Kamiyama A: Antigenicity of the proteins in soy lecithin and soy oil in soybean allergy. Clinical and Experimental Allergy 1998; 28(12): 1559-1564. https://doi.org/10.1046/j.1365-2222.1998.00431.x

12. Müller $U$, Weber W, Hoffmann A, Franke S, Lange R, Vieths S: Commercial soybean lecithins: a source of hidden allergens? Zietschrift für Lebensmitteluntersuchung und Forschung A 1998; 207: 341-351. https://doi.org/10.1007/s002170050343

13. Ibarguren $M$, López DJ, Escribá PV: The effect of natural and synthetic fatty acids on membrane structure, microdomain organization, cellular functions and human health. Biochimica et Biophysica Acta Biomembranes 2014; 1838: 1518-1528. http://dx.doi.org/10.1016/j.bbamem.2013.12.021

14. Kroenke K, Wood DR, Mangelsdorff AD, Meiero NJ, Powell JB: Chronic fatigue in primary care. Prevalence, patient characteristics, and utcome. Journal of the American Medical Association 1988; 260: 929-934. http://dx.doi.org/10.1001/jama.1988.0341007005702 $\underline{8}$

15. Filler K, Lyon D, Bennett J, McCain N, Elswick R, Lukkahatai N, Saligan LN: Association of mitochondrial dysfunction and fatigue: a review of the literature. BBA Clinical 2014; 1: 12-23. https://doi.org/10.1016/j.bbacli.2014.04.001

16. Ellithorpe RA, Settineri $R$, Mitchell $C A$, Jacques $B$, Ellithorpe $E$, Nicolson GL: Lipid replacement therapy drink containing a glycophospholipid formulation 
rapidly and significantly reduces fatigue while improving energy and mental clarity. Functional Foods in Health and Disease 2011; 1(8): 245-254. DOI: 10.31989/ffhd.v1i8.126

17. Nicolson $G L$, Settineri $R$, Ferreira $G$, Breeding $P$ : Reduction of pain, fatigue, gastrointestinal and other symptoms and improvement in quality of life indicators in fibromyalgia patients with Membrane Lipid Replacement glycerolphospholipids and controlled-release caffeine. International Journal of Clinical Medicine 2018; 9: 560-579. DOI: $10.4236 / \mathrm{ijcm} .2018 .97051$

18. Nicolson GL, Breeding PC: Membrane Lipid Replacement for reduction of pain, fatigue, gastrointestinal and other symptoms in patients with peripheral pain: case reports. Case Reports \& Reviews 2020; 1(2): 1-3. DOI: $10.33425 / 2693-1516.1007$
19. Campbell JN, Meyer RA: Mechanisms of neuropathic pain. Neuron 2006; 52(1): 77-96.

https://doi.org/10.1016/j.neuron.2006.09.021

20. Staud R: Peripheral pain mechanisms in chronic widespread pain. Best Practice Research in Clinical Rheumatology 2011; 25: 155-164. https://doi.org/10.1016/j.berh.2010.01.010

21. Pandey NR, Sparks DL: Phospholipids as cardiovascular therapeutics. Current Opinion in Investigative Drugs 2008; 9(3): 281-285.

https://www.researchgate.net/publication/5540018 P hospholipids as cardiovascular therapeutics

22. U.S. Federal Drug Administration. Agency Response Letter GRAS Notice. Soybean derived lecithins. GRAS Report 2014; GRN000534. https://wayback.archiveit.org/7993/20171031003514/https://www.fda.gov/Fo od/IngredientsPackagingLabeling/GRAS/Noticelnvento ry/ucm431750.htm 